\title{
Cancer Foraging Ecology: Diet Choice, Patch Use, and Habitat Selection of Cancer Cells
}

\author{
Sarah R. Amend ${ }^{1} \cdot$ Robert A. Gatenby ${ }^{2} \cdot$ Kenneth J. Pienta ${ }^{1} \cdot$ Joel S. Brown ${ }^{2}$ \\ Published online: 27 November 2018 \\ (C) Springer Science+Business Media, LLC, part of Springer Nature 2018
}

\begin{abstract}
Purpose of Review Here we connect theories of diet choice, patch use, and habitat selection with cancer biology. Key and only partially answered questions include: Do cancer cells' uptake of nutrients conform to theory? What are the supply and total mass of resources within tumors? Can cancer cell foraging strategies provide indicators for tumor dynamics and therapies? We advocate for a new research subdiscipline of cancer foraging ecology.

Recent Findings Foraging ecology studies feeding behaviors of organisms as adaptations. Virtually all of life exhibits adaptations relating to diet, patch use, and habitat selection. Cancer cells likely exhibit selective nutrient uptake (diet), local depletion of resources (patch use), and motility (habitat selection). In fact, the evolution of adaptive feeding strategies by cancer cells may be an additional hallmark of cancer. In aggregate, the feeding behaviors of cancer cells can be devastating - acidosis, hypoxia, cachexia, necrosis, tissue invasion, and metastasis. While these are well known, little is known regarding the nutrient uptake strategies of individual cancer cells. Foraging theory provides a strong theoretical basis for anticipating what cancer cells might do and how research on cancer foraging ecology — with impact on metastasis research and therapeutic intervention - should proceed.

Summary Normal cells, as "servants" to the whole organism, should not conform to the principles of optimal foraging theory. Cancer cells in response to fluctuating resource supplies, nutrient limitations, and hazards should evolve resource acquisition strategies that are more optimal-foraging-like. Two areas of research make cancer foraging ecology a particularly propitious emerging field. From behavioral and evolutionary ecology, there is a well-developed body of theory suggesting how organisms, including cancer cells, should forage. From cancer cell metabolomics there is a large body of knowledge regarding how cancer cells process and utilize different nutrients as fuel, material, buffers and messenger molecules. We suggest the time is ripe for conjoining foraging ecology with cancer cell metabolomics.
\end{abstract}

Keywords Cancer $\cdot$ Foraging ecology $\cdot$ Consumer-resource dynamics $\cdot$ Tumor ecosystems $\cdot$ Diet choice $\cdot$ Patch use $\cdot$ Habitat selection $\cdot$ Metabolomics

\section{Introduction}

Cancer cells, as units of selection, are subject to the forces of natural selection. Cancer cells, like all other living organisms,

This article is part of the Topical Collection on The Evolutionary and Ecological Pathology of Cancer

Joel S. Brown

squirrel@darwiniandynamics.org

1 The James Buchanan Brady Urological Institute, Johns Hopkins University School of Medicine, Baltimore, MD, USA

2 Department of Integrated Mathematical Oncology, Moffitt Cancer Center, Tampa, FL, USA evolve over time based on pressures placed on them due to the quality of their environment. For example, cancer cells require nutrients and resources for homeostasis, survival, growth, and proliferation. In ecological terms, access to nutrients and resources is described by the science of foraging dynamics. Cancer cells exhibit heritable variation (genetic and epigenetic mutations) and must compete for limited resources, safety, and space, resulting in traits that contribute to more successful heritable phenotypes.

A common limitation of cancer proliferation is substrate availability, which may be disrupted by temporal and spatial fluctuations in blood flow within the disordered structure of the tumor microenvironment, resulting in competition for available resources [1]. As a consequence, cancer cells are under strong selection pressure to evolve foraging 
strategies that allow quick, efficient, and safe resource acquisition [2]. Foraging ecology is the study of feeding behaviors and resource acquisition as adaptations. Within cancers, we view foraging ecology as the interplay between how individual cancer cells respond to resource availabilities and how the collective resource uptake of all of cellular populations within a tumor influences resource availabilities (Table 1).

There is a rich literature focused on the metabolism of cancer, including the utilized nutrients, the mechanisms of consumption, and the dependencies of cancer cells on various resources and the relationship of all of these to survival and proliferation [3]. Indeed, this topic is experiencing a renewed appreciation in recent years as new technologies allow for the specific interrogation of cell-metabolite interaction in addition to metabolism of these resources (reviewed in $[4 \cdot \bullet, 5]$ ).

Here, we review current knowledge on the sources, types, and abundance of resources used by cancer cells. We consider the uptake mechanisms utilized by cancer cells and the nutritional relationships of resources consumed by cancer cells (Table 2). In particular, we apply the concept of foraging theory to expand the current field of cancer metabolism by highlighting previously unidentified questions. The foraging ecology of cancer cells has relevance for targets of therapy and as assessments of neoplasms such as the recently proposed evo- and eco- indices for characterizing cancers where measures of resource availability and utilization provide one axis of the eco-index $[6 \bullet \bullet$.

\section{Foraging Theory}

Feeding behaviors occur at three spatial scales. The smallest is diet choice. Most organisms exist in an ecology with a number of potential sources of nutrients. Diet choice considers the dietary patterns of each organism within this range of options [7]. Can cancer cells adaptively modulate their resource uptake rates? What dynamics promote or constrain these dietary variations? The next scale is patch use, which is the extent to which organisms locally deplete the availability of resources as they feed [8]. We explore the extent to which cancer cells can selectivity cease harvesting particular resources when they become scarce. The largest spatial scale is habitat selection. Habitat selection considers the ability of organisms to move by responding to resource availabilities (the focus of this manuscript), hazards such as predation risk, and the density of competitors [9]. Interestingly, cancer cells have the ability to engineer their own habitat, and therefore available resources, both through promoting local angiogenesis and recruitment of host

Table 1 Foraging ecology terms and definitions

\begin{tabular}{|c|c|c|}
\hline Term & Definition & Units \\
\hline$F$ & Fitness; the proliferation rate minus the death rate & - Units per time \\
\hline$x$ & Population size of cancer cells & $\begin{array}{l}\text { - The total number of cells (i.e., whole tumor burden) } \\
\text { - Density of cells per unit area or volume of the tumor }\end{array}$ \\
\hline$d x / d t$ & $\begin{array}{l}\text { Rate of change in the population size of cancer cells per unit time: } \\
\qquad d x / d t=x F\end{array}$ & \\
\hline$t$ & Time & - Seconds, minutes, hours, or days \\
\hline$R_{i}$ & Abundance of resource $i$ & - Molecules, moles, or mass per unit volume of intracellular fluid \\
\hline$e_{i}$ & Nutritional value of resource $i$ & $\begin{array}{l}\text { - Relative value to other resources (per molecule, mole, or mass) } \\
\text { - Overall contribution to the cell's metabolic needs } \\
\text { (ATP or molecular building block per unit resource) } \\
\text { - Contribution to fitness }\left(e_{i}=d F / d C_{i}\right)\end{array}$ \\
\hline$C_{i}$ & Consumption rate of resource i by a single cell & - Molecules, mass, or moles per unit time \\
\hline$k_{i}$ & $\begin{array}{l}\text { Fixed need for resource } i \\
\text { The cell should modulate consumption to meet this fixed demand: } \\
\qquad C_{i}=k_{i}\end{array}$ & - Resource per unit time \\
\hline$C_{i}\left(R_{i}\right)$ & $\begin{array}{l}\text { Functional response to resource } i \\
\text { Usually } C_{i} \text { will be an increasing function of resource availability } R_{i} \text {. }\end{array}$ & \\
\hline$a_{i}$ & $\begin{array}{l}\text { Fraction of available resources harvested } \\
\text { If this is the only limit on consumption then it will increase linearly } \\
\text { with resource concentration: } \\
C_{i}\left(R_{i}\right)=a_{i} R_{i}\end{array}$ & - Resource per unit time \\
\hline$h_{i}$ & $\begin{array}{l}\text { The time it takes for a cancer cell to handle a unit of resource } i \text { during } \\
\text { which time it is not possible to further uptake more of that resource. } \\
\text { If this is the only limit on consumption, then it will increase with } \\
\text { abundance but at a decelerating rate: } C_{i}\left(R_{i}\right)=a_{i} R_{i} /\left(1+a_{i} h_{i} R_{i}\right)\end{array}$ & - Time \\
\hline
\end{tabular}


Table 2 Nutritional relationships of resources

\begin{tabular}{lll}
\hline Resource relationship & $F$ is proportional to & Definition \\
\hline $\begin{array}{l}\text { Perfectly substitutable } \\
\text { Complementary }\end{array}$ & $\begin{array}{l}e_{1} C_{1}+e_{2} C_{2} \\
e_{1} e_{2}\left(C_{1}\right)^{\alpha}\left(C_{2}\right)^{\beta}\end{array}$ & $\begin{array}{c}\text { The fitness value of consuming resource } 1 \text { and resource } 2 \text { is equal. } \\
\text { The fitness value of consuming resource } 1 \text { declines as more is consumed (diminishing returns) } \\
\text { The fitness value of consuming resource } 2 \text { increases with the consumption of resource } 1 \\
\text { (better to consume a mix of both than just one resource) }\end{array}$ \\
& $\begin{array}{c}0<\alpha, \beta<1 \text { are scaling factors that scale the degree of diminishing returns to consuming more of resource } 1 \text { and } 2, \\
\text { respectively } \\
\min \left\{e_{1} C_{1}, e_{2} C_{2}\right\}\end{array}$ & $\begin{array}{c}\text { The consumption of one resource or the other determines fitness; increased uptake of the } \\
\text { non-limiting resource has no positive effect on the cell's fitness }\end{array}$ \\
\hline
\end{tabular}

microenvironment cells (M2 macrophages, cancer associated fibroblasts, etc.) $[10,11]$.

\section{Resources}

We define a resource as any molecule (inorganic or organic and micro- or macromolecules) that can be taken up by all or a subset of cancer cells and used to promote homeostasis, growth, and/or survival [12]. Resources generally provide material or fuel [3]. The acquisition of a resource means that it is temporarily (e.g., potassium ions) or permanently (e.g., oxygen) used up. Glucose, glutamine, and other amino acids make up $10-15 \%, 10 \%$, and $20-40 \%$ of the carbon in a cancer cell, respectively [13•]. A resource such as glucose serves primarily as fuel to make ATP and drive cell metabolism. In contrast, a resource such as glutamine has many roles: it can be used as a building block in polypeptides and nucleotides, metabolized as fuel, or broken down and reconfigured into other amino acids or even nucleotides [14•]. Similarly, fatty acids can be used to produce structures (cell membranes), provide fuel, and be transformed into hormones, growth factors, and diverse molecules [15]. Macromolecules may be usable directly for structures and enzymes (albumin and lipases) or be broken down to provide molecular building blocks and fuel. Taken together, these observations demonstrate that eukaryotic cells, and cancer cells in particular, have multiple and diverse pathways for acquiring the necessary substrate for survival and proliferation [16, 17].

Growth factors are a special class of resources. They do not seem to be directly useful as building blocks, structures, or fuel, yet growth factors, such as estrogens and androgens, may be critical controllers of tissue specific cell division or activity [3]. They can be produced by specialized cells at distant location in the body (e.g., testes, ovaries) and then distributed through the vasculature so that local concentrations in tumors are in part governed by local blood flow. Other growth factors are produced locally by fibroblasts, macrophages, and other host cells within the tumor microenvironment and made available to a smaller neighborhood of cancer cells. In both cases, growth factors act as resources to govern survival, proliferation, and movement of specific types of cells, and some growth factors may regulate other nutrient consumption. Given their non-essential contribution to material and fuel, however, it is unsurprising that cancer cells often evolve the ability to produce their own hormones and growth factors or reconfigure vital metabolic pathways to be independent of these molecules [18].

\section{Nutritional Relationships}

Diverse sets of nutrients are required for the survival and proliferation of cancer cells, measured as the expected net proliferation rate of a cancer cell lineage, $F$. If $x$ is the population size of the lineage, the population size over time $=d x / d t=x F$. What is less well known is how various combinations of resource uptake influence net proliferation $(F)$. For instance, we can let $\boldsymbol{C}=\left(C_{1}, \ldots C_{n}\right)$ be the consumption rate of a cell on $n$ different resources. Net proliferation rates will be influenced by this consumption rate $(C)$, and in most cases, consuming more of a resource will generally improve, or at least not hurt, $F[19]$.

In addition to abundance, the value and purpose of a resource are also critical. Two resources are substitutable if their value to the cancer cell is a weighted average of their consumption rates: $F$ is a function of $e_{1} C_{1}+e_{2} C_{2}$ where $e_{1}$ and $e_{2}$ are the relative values of the two resources (e.g., glucose and fructose). Two resources are complementary if their relative value to the cancer cell changes with consumption (e.g., glucose and cysteine). Increasing consumption of one makes the other relatively more valuable: $F$ may be a function of $e_{1} C^{\alpha}{ }_{1}+e_{2} C^{\beta}{ }_{2}$ or $e_{1} e_{2} C_{1}{ }^{\alpha} C^{\beta}{ }_{2}$ where $\alpha$ and $\beta$, scaling factors representing the respective value of the resource, are greater than zero but less than one. Thus, there are diminishing returns to consuming more of a given resource. Both glucose and glutamine (or cysteine) can be fuel or material, but glucose serves best for fuel and glutamine (or cysteine) for material. Fatty acids may enhance the utilization and metabolism of glucose and glutamine [20]. Two resources are essential if their relative value to the cancer cell is based on whichever one is most limiting (Liebig's Law of the Minimum): $F$ is a function of the minimum of $e_{1} C_{1}$ and $e_{2} C_{2}$. Essential 
resources for cancer may include oxygen, various amino acids, growth factors, and ions.

\section{Resource Uptake and Diet Choice}

Cancer cells can take up resources through multiple mechanisms, including diffusion, active transport, and micropinocytosis (Fig. 1). Diffusion allows the movement of molecules in and out of the cell as determined by the relative concentration on either side of the plasma membrane. Passive diffusion is restricted to small uncharged hydrophobic molecules that can dissolve across the phospholipid bilayer. Facilitated diffusion, while still governed by the relative concentration of the resource, requires carriers or channel proteins that allow larger and/or charged molecules to cross the plasma membrane (e.g., amino acids, ions, carbohydrates), thus introducing a "handling cost" to consumption. Active transport enables the cell to move a molecule up its concentration gradient by using energy such as hydrolysis of ATP or another coupled reaction (e.g., ion pumps: $\mathrm{Na}^{+}-\mathrm{K}^{+}, \mathrm{Ca}^{++}$; $\mathrm{ABC}$ transporters: carbohydrates, amino acids). Other molecules are taken up by the cell via receptor-mediated endocytosis in which the molecule binds its receptor on the cell surface and a vesicle is formed by the inward budding of the plasma membrane into the cell (e.g., hormones, growth factors). In contrast with the transport of specific nutrients via receptor-mediated endocytosis, a cell can engulf adjacent material non-selectively via the endocytic process of micropinocytosis (or, for larger molecules, macropinocytosis).

The functional response, $C_{i}(R)$, is a foraging ecology concept that describes the rate of resource uptake as a function of resource availabilities: $R=\left(R_{1}, \ldots R_{n}\right)$. It describes how quickly a cancer cell acquires a resource and how this uptake is influenced by the concentration of resources in their environment. This rate of resource acquisition supports fuel for metabolic activity and materials for maintenance, growth, and proliferation. The functional response relates to how quickly a cell or collection of cells will deplete resources. In terms of resource competition, natural selection will favor both speed and efficiency of nutrient uptake [21].

If resource uptake is entirely driven by the needs of the cell and is not constrained by resource availability, then $C_{i}=k_{i}$, a constant that describes resource requirement. For instance, oxygen is only useful for oxidative phosphorylation. If available carbon substrates such as glucose, pyruvates, or lactates are available for generating ATP in the mitochondria, then oxygen uptake should simply match this need. The Warburg effect recognizes continued anaerobic glycolytic metabolism despite the presence (or the absence) of ample oxygen [22, 23].

For resources that passively diffuse into the cell, or perhaps via facilitated diffusion, the rate of resource uptake will be linearly related to availability: $C_{i}=a_{i} R_{i}$ where $a_{i}$ is the uptake rate of the cell per unit resource. True passive diffusion across the phospholipid membrane is rare and restricted to gasses and small hydrophobic molecules (e.g., oxygen, water, ethanol). Facilitated diffusion by carrier proteins and channel proteins is common and is the route of transport for glucose and other sugars, amino acids, and nucleotides. Evidence suggests that the rate of glucose consumption and uptake is directly related to its availability [24, 25].

When resources are acquired through facilitated diffusion or active transport, or engage an extracellular receptor, there may be some maximal rate resource uptake based on channels, pores, or transporter molecules [26]. Glucose requires a carrier protein (e.g., GLUT-1) that binds to extracellular glucose. Upon binding, the carrier protein changes conformation and rotates, releasing the glucose into the cell, and then reverts to its original conformation. Thus, each carrier protein experiences a handling time and cost. Having more transporter molecules will increase the rate of glucose uptake by increasing $a_{i}$, but the cell's functional response on glucose will eventually saturate for any given number of transporters. Such a functional response may take the form of $C_{i}=a_{i} R_{i} /\left(1+a_{i} h_{i} R_{i}\right)$ where $h_{i}$ represents a handling time or transportation cost to taking up the resource. With such a functional response, uptake rates reach a maximum value of $1 / h_{i}$ as resource availability increases to extremely high concentrations. Such a functional response is likely the norm for most resources, though actual measurements are rare.

Cancer cells can likely direct their uptake towards particularly valued or rare nutrients by upregulating transporters. This can lead to a functional response in which uptake accelerates with resource concentration but exhibits a diminishing rate of increase at higher concentrations: $C_{i}=a_{i} R^{\beta}{ }_{i} /(1+$ $a_{i} h_{i} R^{\beta}{ }_{i}$ ) where the scaling factor $\beta>1$. For example, because of their increased proliferation and protein synthesis, cancer cells have a high amino acid demand. In order to consume adequate levels of amino acids, cancer cells must upregulate one or more (but not all) amino acid transporters [27•]. The cost of resistance in a class of cancer cells resulted from increased glutamine consumption and metabolism to maintain redox homeostasis within the cell $[28 \bullet \bullet]$.

Based on these functional responses, can cancer cells exhibit biased or selective resource uptake? Foraging theory makes a number of predictions regarding selective feeding by cancer cells. When overall resource availabilities are high, cancer cells should be more selective and reject resource items of lower value. Resources, in general, will be ranked according to their value divided by the effort required to acquire them: $e_{i} / h_{i}$.

It is likely that those resources consumed by passive transport (e.g., oxygen) are not governed by selective resource uptake: what is available to be consumed will be taken up until equilibrium is reached. In contrast, when active mechanisms of resource uptake and consumption require energy, 


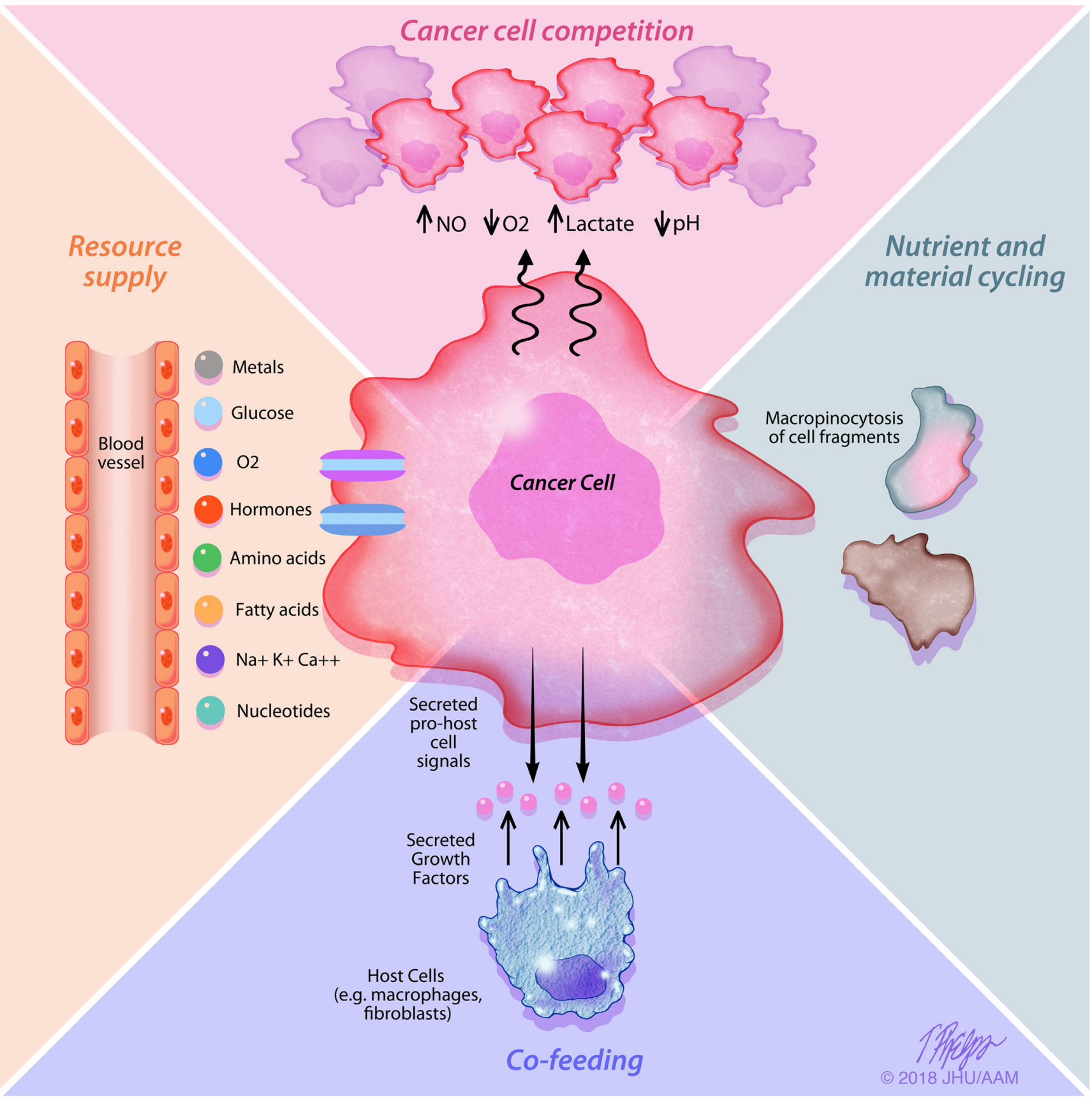

Fig. 1 Mechanisms of resource uptake by the cancer cell based on foraging substrate. The cancer cell can consume resources via multiple different mechanisms, dependent on resource availability and diet choice. Constant resource supply: Resources are provided by a relatively steady resource supply (e.g., blood vessel), and the cell consumes the resources via passive diffusion, active diffusion, and active transport (left). Cofeeding of cancer cells and host cells: cancer cells have the capacity to engineer their own habitat via recruitment of host cells that in turn provide growth factors and other nutrients for the local habitat (bottom). Nutrient

selective resource uptake would be adaptive. In normal cells, there is evidence of nutrient uptake preference, mediated by nutrient sensors and growth factor signaling. For example, growth factor-mediated PI3K/Akt signaling mediates and material recycling: cancer cells engulf neighboring dying cells or cell debris in a non-selective manner to consume macromolecules, proteins, lipids, etc. (right). Cancer cell competition: an individual cancer cell participates in and is influenced by the metabolic waste of a growing tumor mass (top); this waste can decrease the activity of nutrient transporters (e.g., glucose transporters have decreased activity in acidic conditions) and also be used as a substrate itself (e.g., lactate can be used as an energy source)

translocation of glucose transporters to the cell membrane to facilitate glucose uptake [29-31] (reviewed in [5]). Upon oncogenic transformation, selective resource uptake mediated by growth factor binding may be uncoupled, resulting in 
constitutive glucose uptake $[5,18,22]$. There is similar evidence for the constitutive uptake of glutamine and essential amino acids in order to support the high proliferation rates of cancer cells.

For a highly proliferative cancer cell, it is likely that the value of substrates for production of ATP and as material for cell growth is so high as to outweigh the costs of acquisition. Therefore, it is likely that partial preferences for nutrients would be the norm for essential and complementary resources. It is also possible that mutations in a different set of oncogenes and tumor suppressor genes would lead to preferential uptake of resources based on transporter activation. In this case, preference may depend on the effort and cost of acquisition. In contrast, if a resource is needed to activate a constitutive pathway, then need may drive consumption more than availability. For example, breast cancer cells may simply increase the number of estrogen receptors in response to estrogen suppression therapies or reroute metabolic pathways to become entirely independent of the otherwise essential resource of estrogen.

The presence of one resource may strongly influence the uptake of another. Glutamine consumption enhances uptake of the essential amino acid leucine (among other amino acids) through LAT1 $[32,33]$. In normal cells, glucose consumption via GLUT1 is regulated by growth factor binding such that when growth factors are absent, glucose consumption is reduced, despite high extracellular glucose levels [29]. Such adjustments may be in response to the resource being complementary or essential. If one nutrient is mostly limiting, then there is little point in harvesting other less limiting ones.

The availability of one resource influencing the uptake and/ or value of another may be manifestation of what has been called "metabolism addiction." For instance, in cancer, glucose and/or glutamine uptake may be decoupled from associated complementary resources such that uptake is only constrained by availability (reviewed in $[4 \bullet \cdot]$ ). In cells that are glutamine-addicted, however, amplification of c-myc increases expression of glutamine transporters as well as glutamine-utilizing enzymes, thus increasing glutamine consumption and conversion to glutamate [34]. Notably, this accumulation of glutamate initiates a complementary resource relationship: glutamate participation in the TCA cycle promotes cysteine consumption [35].

\section{Resource Depletion and Optimal Patch Use}

Microenvironment heterogeneity is a characteristic of tumors. Temporal and spatial variations in vasculature, local geometry of the tumor, and the structure of normal cells and stroma within the tumor generate a patchiness of distribution and abundance of nutrients [36, 37]. Neighborhoods of cancer cells likely experience periods and places with high- or lowresource supply [12]. Resources are supplied through the vasculature, the extracellular space (e.g., extracellular matrix), local host microenvironment cells, and local cancer cells (e.g., waste product lactate then consumed as an energy source; growth factors), and the scavenging on material released from dead or dying cells [38, 39] (Fig. 1).

Resource-rich and resource-poor regions vary over time as cancer and microenvironment cells consume the resources that are then replenished with varying rates $[40 \bullet 0$. Some areas may experience a relatively stable supply of continuously renewed resources (e.g., adjacent to blood vessels) while other areas may ebb over time as the resources are consumed (e.g., from tumor microenvironment cells). Regardless of the consistency of the resource supply to these patches, the richness of a patch will necessarily change over time as cells consume resources and deplete the patch, while simultaneously cells move into and out of the patch, either through cell movement or through the proliferation of new cells. Beyond simply rich or poor, patches may supply different combinations of resources. For instance, a patch may contain resources such as sugars and growth factors, while supplying very little of other resources such as oxygen. As such, a cell's foraging strategy must be dynamic, necessarily varying from patch-to-patch and moment-to-moment. Ultimately, the resources within a patch represent a dynamic balance between renewal and depletion.

When foraging in a patch, an individual expends energy to consume resources (search and handling) and gains benefit from the resources. While depleting a patch, the individual will experience diminishing returns as the energy expended to consume resources exceeds the benefit gained. This phenomenon then requires that, for optimal patch use, not all resources are consumed - those resources that are not "worth it" to the individual. A Giving Up Density (GUD) describes the level of resources that are abandoned in the patch by a foraging individual $[2,41]$. This "giving up" may take form in two ways: (1) the cell moves from that patch in search of another higher-resource patch or (2) the cell stops foraging and remains stationary, reducing its metabolism and waits for the patch to replenish. An old study suggested that glutamine uptake by cancer cells ceases when arterial concentrations are less than $0.5 \mathrm{mM}$ [42]. Understanding the GUD of a particular subset of cancer cells and how the GUD changes with diet choice and mechanisms of resource uptake would provide a wealth of information to enable predictions of behavior, specifically cell movement, invasion, and persistence, under varying resource availabilities.

There is some incidental experimental evidence of the foraging behavior of cancer cells, but there is little research specifically interrogating these questions. Indeed, it is unknown whether cancer cells have GUDs or if they completely deplete their environment of all resources. It is known that the abundance of glucose within a tumor is just 10-33\% that of normal tissue [43]. Remarkably, even the relatively elementary (though experimentally challenging) questions of the flow of nutrients in and out of a tumor or total measures of nutrient 
availabilities present in a tumor remain unanswered (but see [39]). It is unclear whether cancer cells need more (or higherquality or greater diversity of) or fewer (or lower-quality and less variety of) resources compared to their normal cell counterparts. Normal cells bear the cost of their differentiated activities but typically do not proliferate as frequently as their malignant counterparts. In contrast, cancer cells must replicate their internal structure to permit replication but will devote far fewer resources to carry out functions that would ordinarily benefit the host multicellular organism. Interestingly, the energy and substrate (carbon, nitrogen, phosphate, etc.) budgets of normal or cancer cells in vivo are largely unknown. Decades of experience with FdG PET scanning in clinical cancers demonstrate that most malignant cells have increased glucose uptake compared to normal cells, but these observations have not been integrated into a comprehensive understanding of the differences of the overall resource demand and utilization budgets in malignant and normal mammalian cells.

While these quantifications would be most valuable in the setting of a human tumor, in vitro culturing techniques provide the most insight and a first-step approximation of the presence of a cancer cell GUD. Determining the minimum resource requirements of a cancer cell has been a critical for cell biologists since the advent of in vitro culturing techniques. Indeed, early work by Harry Eagle, George Gey, and others demonstrates the minimum media requirements for subculturing of the HeLa cancer cell line and provided the first glimpse into the nutrient uptake — or foraging — of cancer cells [44]. Even these careful formulations, however, provide all nutrients, including sugars and essential amino acids, in excess and include the essential, but highly variable and largely unquantifiable, nutrient supplement of animal serum (e.g., fetal bovine serum, FBS).

Opposite excess resources is starvation, a standard laboratory procedure to synchronize in vitro cultures by inducing $\mathrm{G} 0 / \mathrm{G} 1$ cell cycle arrest. Rather than determining a titrated system, however, most serum starvation procedures immediately transition cultures from 10 to $20 \%$ FBS to $0-1 \%$ FBS [45]. Though these preparatory steps do not identify a cancer cell population's GUD (though measuring the nutrients from starved cells when they undergo growth arrest may shed light on the topic), serum starvation does indicate that there is a point at which cells decrease metabolism and proliferation - in essence, they stop foraging. Beyond the milieu of cell-supportive factors present in FBS, many groups have also demonstrated that effect sizes are amplified upon culturing cancer cells in low sugar or low oxygen, likely challenging the cancer cells to the limit of or beyond their GUD to observe a response.

It is possible - perhaps even likely, given the aberrant nature of cancer cells - that, while normal cells may have a GUD at which the cells undergo senescence or apoptosis, cancer cells by nature of their altered metabolism and immortality have lost the ability to sense and respond to a GUD. In very low nutrient levels, such as in low-oxygen and low $\mathrm{pH}$ regions of a tumor, cancer cells undergo autophagy. Autophagy is a short-term solution to promote immediate survival of a cell by self-digestion of organelles as an energy source. If a lack of resources continues, the cell dies [46]. That autophagy exists at all may indicate that the cancer cell failed to stop foraging at its GUD, resulting in severe energy restriction and subsequent activation of type II programmed cell death.

\section{Habitat Selection and Cell Movement}

In nature, most organisms will select their habitats in a manner that improves their fitness, either based on safety, survival costs, and/or resource availabilities. The movement of individuals away from less favorable places to more favorable ones means that, overall, individuals will become distributed in space in a manner that roughly equalizes opportunities. By moving from a place, an individual makes the habitat better for those that remain (more food and space per individual). By moving into a place, the habitat must now support an additional individual. Within a habitat, resource limitation means that fitness (i.e., net proliferation rate) declines with the number of individuals within that habitat: $d F_{i} / d x_{i}<0$ for some habitat $i$. If individuals can move freely, share equally in the opportunities of a habitat, and have perfect information on the habitats, then individuals should distribute themselves among habitats so that fitness are equal in an ideal free distribution: $F\left(x_{1}\right)=F\left(x_{2}\right)=\ldots F\left(x_{\mathrm{n}}\right)$ where there are $n$ habitats.

Cancer cells likely cannot achieve such a distribution. They may lack sufficient motility, sensory capacity, and information, but any capacity to move from poorer habitats into better habitats will be revealing. For instance, high glycolytic activity is associated with motility in prostate cancer cells [47•]. Cell density within particular habitats should correlate with nutrient supply. At very local scales, there may be a declining density of cancer cells with distance from vasculature [48]. Regions of necrosis indicate a paucity of nutrients and perhaps an accumulation of toxins. Standard in vitro migration and invasion assays demonstrate the capacity of cancer cells for this type of habitat selection (e.g., moving towards higher nutrients: FBS, growth factors). Measures of cancer cell densities may provide insights into or act as a surrogate measure of, resource supply and habitat favorability from the perspective of the cancer cell [49]. In glioblastoma patients, T1 gadolinium, FLAIR, and T2 gadolinium MRI has been used to identify distinct tumor habitats [50]. A high frequency of resource availability ${ }^{\text {high }}$-cancer cell density ${ }^{\text {low }}$ habitats was a predictor of better patient outcome compared to other habitat classifications. Glioblastoma cells are known to be particularly motile lending support that the distribution of cells across habitat types may include forms of habitat selection 
in response to nutrients, risks, and competition from other cells [51].

\section{Conclusions}

Cancer cells are divorced from their normal cell counterpart's function and are no longer restrained by body-wide homeostatic requirements. In contrast, cancer cell fitness (proliferation and population size) is severely limited by resource availability within the heterogeneous and highly competitive local habitat of the microenvironment. These cancer cells should conform to the expectations of foraging theory: diet choice, patch use, and habitat selection.

To survive under the strong selective pressures in a tumor, cancer cells must evolve foraging strategies to maximize their fitness given the circumstances of resource scarcity. Cancer cells should become more selective and effective in their harvest of particular nutrients. This diet choice should reflect the specific nutritional values of each resource and the nutritional relationships between resources. Cancer cells should evolve patch use behaviors where they deplete nutrients only to point where additional uptake rates compensate for the uptake costs (giving-up-density). Finally, motile cancer cells demonstrate habitat selection. Simple motility may move cancer cells from crowded to less crowded regions: away from nutrient depleted regions and/ or towards nutrient rich areas. In short, a hallmark of cancer may be the evolution of foraging behaviors that conform to the expectations of foraging theory $[4 \bullet \bullet, 52-54]$. The study of such uptake behaviors-partially selectivities in diet, functional response curves, giving-up densities, and habitat selection - is in its infancy and is often overlooked by cancer biologists. Yet, in other fields of ecology and conservation feeding behaviors provide valuable indicators [55••].

The aggregate feeding behaviors of cancer cells have demonstrable and clinically important consequences for tumor properties, responses to therapy, and patient outcome. These are well known and include $\mathrm{pH}$, hypoxia, microenvironment heterogeneity, upregulation of transporters, necrosis, epithelial to mesenchymal transformation, invasion, and metastasis. Less well known are the actual foraging behaviors of the individual cells that result in these emergent properties. We feel the time is propitious for the new subdiscipline of cancer foraging ecology.

Acknowledgments We thank Chris Whelan and Mark Lloyd with insightful discussion and pointing us to important papers in the literature.

Funding Information SRA is supported by American Cancer Society Postdoctoral Fellowship PF-16-025-01-CSM, a Prostate Cancer Foundation Young Investigator award and Patrick C. Walsh Prostate Cancer Research Award; KJP is supported by the NIH/NCI (CA093900 and U54CA210173) and the Prostate Cancer Foundation. JSB and RAG were supported by the European Union's Horizon 2020 research and innovation program (Marie Sklodowska-Curie grant agreement no. 690817), the James S. McDonnell Foundation grant, "Cancer therapy: Perturbing a complex adaptive system," a V Foundation grant, NIH/ National Cancer Institute (NCI) R01CA170595, Application of Evolutionary Principles to Maintain Cancer Control (PQ21), and NIH/ NCI U54CA143970-05 [Physical Science Oncology Network (PSON)] "Cancer as a complex adaptive system."

\section{Compliance with Ethical Standards}

Conflict of Interest Sarah R. Amend, Robert A. Gatenby, Kenneth J. Pienta, and Joel S. Brown declare that they have no conflict of interest.

Human and Animal Rights and Informed Consent This review did not generate, use, or analyze any primary data with human or animal subjects.

\section{References}

Papers of particular interest, published recently, have been highlighted as:

- Of importance

- Of major importance

1. Gillies RJ, Brown JS, Anderson ARA, Gatenby RA. Evo-evolutionary causes and consequences of temporal variations in intratumoral blood flow. Nat Rev Cancer. 2018;18(9):576-85

2. Brown JS. Vigilance, patch use and habitat selection: foraging under predation risk. Evol Ecol Res. 1999;1(1):49-71.

3. Thompson CB. Rethinking the regulation of cellular metabolism. Cold Spring Harb Symp Quant Biol. 2011;76:23-9.

4.• Pavlova NN, Thompson CB. The emerging hallmarks of cancer metabolism. Cell Metab. 2016;23(1):27-47. The authors recognize that cancer cells are under selection to acquire nutrients quickly, efficiently and in novel ways. All of this enhances survival and proliferation under conditions of severe nutrient limitation. Hence, they propose a relationship between six hallmarks and metabolic reprogamming: (1) 'deregulated uptake of glucose and amino acids"' (2) "use of opportunistic modes of nutrient acquisition", (3) "use of glycolysis/TCA cycle intermediates for biosynthesis and NADPH production", (4) "increased demand for nitrogen" [or phosphorus?], (5) "alterations in metabolite-driven gene regulation", and (6) "metabolic interactions with the microenvironment". We see these evolving traits as of the mechanisms propelling cancer cells towards optimal foraging.

5. Palm W, Thompson CB. Nutrient acquisition strategies of mammalian cells. Nature. 2017;546(7657):234-42.

6.• Maley CC, Aktipis A, Graham TA, Sottoriva A, Boddy AM, Janiszewska M, et al. Classifying the evolutionary and ecological features of neoplasms. Nat Rev Cancer. 2017;17(10):605-19. This consensus paper recognizes the eco-evolutionary dynamics that drive changes in cancer cell numbers, the frequency and coexistence of different cancer cell types within the tumor, metastatic potential, and the evolution of traits that promote survivorship and proliferation under what may be fairly homogeneous or heterogeneous tumors. An Evo-Index is defined based on cell type diversity within the tumor and changes in tumor heterogeneity. An Eco-Index considers both resource availability (food) and hazards (predation) that represent a universal 
property of all ecological systems. The goal of having 16 combinations of low and high Eco- and Evo-indices is to have a straightforward and sound means for clinically scoring cancers. The indices are meant for evaluating prognoses, treatment options and anticipated outcomes.

7. Pulliam HR. On the theory of optimal diets. Am Nat. 1974;108(959):59-74.

8. Charnov EL. Optimal foraging, the marginal value theorem. Theor Popul Biol. 1976;9(2):129-36.

9. Morris DW. Toward an ecological synthesis: a case for habitat selection. Oecologia. 2003;136(1):1-13.

10. Yang KR, Mooney SM, Zarif JC, Coffey DS, Taichman RS, Pienta KJ. Niche inheritance: a cooperative pathway to enhance cancer cell fitness through ecosystem engineering. J Cell Biochem. 2014;115(9):1478-85.

11. Ibrahim-Hashim A, J. Gillies R, S. Brown J, A. Gatenby R. Coevolution of tumor cells and their microenvironment: "niche construction in cancer". In: Ecology and evolution of Cancer. Amsterdam: Elsevier; 2017. p. 111-7.

12. de Groot AE, Roy S, Brown JS, Pienta KJ, Amend SR. Revisiting seed and soil: examining the primary tumor and cancer cell foraging in metastasis. Mol Cancer Res. 2017;15(4):361-70.

13. Hosios AM, Hecht VC, Danai LV, Johnson MO, Rathmell JC, Steinhauser ML, et al. Amino acids rather than glucose account for the majority of cell mass in proliferating mammalian cells. Dev Cell. 2016;36(5):540-9. The authors explore the contribution of consumed resources to cell mass. They find that amino acids, while consumed at lower rates than glucose and glutamine, compose the majority of proliferating cells' carbon mass. Moreover, the authors trace the ultimate fates of consumed nutrients, demonstrating that different, seemingly similar, resources are used for different purposes (e.g., glutamine's role in amino acid biosynthesis).

14. De Vitto H, Perez-Valencia J, Radosevich JA. Glutamine at focus: versatile roles in cancer. Tumor Biol. 2016;37(2):1541-58. In this comprehensive review of glutamine metabolism in cancer, the authors discuss the myriad of roles for glutamine in proliferative cells, including contribution to protein biomass and its role in essential cellular bioenergetics. They also discuss glutamine addiction in oncogene- and tumor suppressor-driven cancer as a possible therapeutic target.

15. Gentric G, Mieulet V, Mechta-Grigoriou F. Heterogeneity in cancer metabolism: new concepts in an old field. Antioxid Redox Signal. 2017;26(9):462-85.

16. Amelio I, Cutruzzolá F, Antonov A, Agostini M, Melino G. Serine and glycine metabolism in cancer. Trends Biochem Sci. 2014;39(4):191-8.

17. Spinelli JB, Yoon H, Ringel AE, Jeanfavre S, Clish CB, Haigis MC. Metabolic recycling of ammonia via glutamate dehydrogenase supports breast cancer biomass. Science. 2017;358(6365):941-6.

18. Hanahan D, Weinberg RA. Hallmarks of cancer: the next generation. Cell. 2011;144(5):646-74.

19. Tilman D. Resource competition and community structure. Princeton: Princeton University Press; 1982.

20. Biswas S, Lunec J, Bartlett K. Non-glucose metabolism in cancer cells-is it all in the fat? Cancer Metastasis Rev. 2012;31(3-4):68998.

21. Brown JS. Coexistence on a seasonal resource. Am Nat. 1989;133(2):168-82.

22. Warburg O. The metabolism of carcinoma cells. J Cancer Res. 1925;9(1):148-63.

23. Gatenby RA, Gillies RJ. Why do cancers have high aerobic glycolysis? Nat Rev Cancer. 2004;4(11):891-9.

24. Gullino PM, Grantham FH, Courtney AH. Glucose consumption by transplanted tumors in vivo. Cancer Res. 1967;27(6):1031-40.
25. Sauer LA, Stayman JW 3rd, Dauchy RT. Amino acid, glucose, and lactic acid utilization in vivo by rat tumors. Cancer Res. 1982;42(10):4090-7.

26. Cooper GM, Hausman R. A molecular approach. In: The Cell. 2nd ed. Sunderland, MA: Sinauer Associates; 2000

27. Bhutia YD, Babu E, Ramachandran S, Ganapathy V. Amino acid transporters in cancer and their relevance to "glutamine addiction": novel targets for the design of a new class of anticancer drugs. Cancer Res. 2015;75(9):1782-8. The authors highlight a possible new class of anti-cancer therapies aimed at exploiting cancer cells' glutamine addiction. Specifically, they discuss preclinical studies inhibiting amino acid transporters SLC1A5, SLC7A5, SLC7A11, and SLC6A14, thus impeding resource consumption and leading to cell death.

28.• Duan G, Shi M, Xie L, Xu M, Wang Y, Yan H, et al. Increased glutamine consumption in cisplatin-resistant cells has a negative impact on cell growth. Sci Rep. 2018;8(1):4067. The authors demonstrate the cost of resistance in three cancer cell lines in which there are Cisplatin resistant and sensitive subclones. The mechanism of resistance appears to by the upregulation of glutamine metabolism in a manner that is inefficient yet protects the cells from antioxidant toxicity. The upregulated metabolism may actually permit faster growth rates but at the cost of equilibrium population sizes. The sensitive cells all have higher carrying capacities. In this example, it appears that the sensitive cells have evolved to be efficient foragers and utilizers of glutamine which is then sacrificed for safety in the face of therapy. Such a cost of resistance is essential in models of adaptive therapy.

29. Rathmell JC, Heiden MGV, Harris MH, Frauwirth KA, Thompson CB. In the absence of extrinsic signals, nutrient utilization by lymphocytes is insufficient to maintain either cell size or viability. Mol Cell. 2000;6(3):683-92.

30. Barthel A, Okino ST, Liao J, Nakatani K, Li J, Whitlock JP Jr, et al. Regulation of GLUT1 gene transcription by the serine/threonine kinase Akt1. J Biol Chem. 1999;274(29):20281-6.

31. Vander Heiden MG, Plas DR, Rathmell JC, Fox CJ, Harris MH, Thompson CB. Growth factors can influence cell growth and survival through effects on glucose metabolism. Mol Cell Biol. 2001;21(17):5899-912.

32. Nicklin P, Bergman P, Zhang B, Triantafellow E, Wang H, Nyfeler $\mathrm{B}$, et al. Bidirectional transport of amino acids regulates $\mathrm{mTOR}$ and autophagy. Cell. 2009;136(3):521-34.

33. Yanagida O, Kanai Y, Chairoungdua A, Kim DK, Segawa H, Nii T, et al. Human L-type amino acid transporter 1 (LAT1): characterization of function and expression in tumor cell lines. Biochim Biophys Acta. 2001;1514(2):291-302.

34. Gao P, Tchernyshyov I, Chang TC, Lee YS, Kita K, Ochi T, et al. cMyc suppression of miR-23a/b enhances mitochondrial glutaminase expression and glutamine metabolism. Nature. 2009;458(7239): $762-5$.

35. Conrad M, Sato H. The oxidative stress-inducible cystine/glutamate antiporter, system x (c) $(-)$ : cystine supplier and beyond. Amino Acids. 2012;42(1):231-46.

36. Lloyd MC, Rejniak KA, Brown JS, Gatenby RA, Minor ES, Bui MM. Pathology to enhance precision medicine in oncology: lessons from landscape ecology. Adv Anat Pathol. 2015;22(4):267-72.

37. Zellmer VR, Zhang S. Evolving concepts of tumor heterogeneity. Cell Biosci. 2014;4(1):69.

38. Jain RK. Determinants of tumor blood flow: a review. Cancer Res. 1988;48(10):2641-58.

39. Vaupel P, Kallinowski F, Okunieff P. Blood flow, oxygen and nutrient supply, and metabolic microenvironment of human tumors: a review. Cancer Res. 1989;49(23):6449-65.

40.• Amend SR, Pienta KJ. Ecology meets cancer biology: the cancer swamp promotes the lethal cancer phenotype. Oncotarget. 2015;6(12):9669-78. The authors introduce the concept of 
cancer-driven ecosystem engineering, autoeutrophication, leading to the formation of a cancer swamp. In particular, the authors highlight the resource poverty and toxin accumulation in a rapidly growing tumor. Importantly, the authors emphasize that a growing and changing tumor, and its associated tumor microenvironment, change over time and with passing generations of cells, thus influencing individual cell phenotype and selecting for more aggressive more motile phenotypes. In addition, the authors discuss how the lethal syndromes that ultimately cause cancer-related deaths are the result of the formation and maintenance of the cancer swamp(s).

41. Brown JS. Patch use as an Indicator of habitat preference, predation risk, and competition. Behav Ecol Sociobiol. 1988;22(1):37-47.

42. Kallinowski F, Runkel S, Fortmeyer HP, Förster H, Vaupel P. Lglutamine: a major substrate for tumor cells in vivo? J Cancer Res Clin Oncol. 1987;113(3):209-15.

43. Birsoy K, Possemato R, Lorbeer FK, Bayraktar EC, Thiru P, Yucel $\mathrm{B}$, et al. Metabolic determinants of cancer cell sensitivity to glucose limitation and biguanides. Nature. 2014;508(7494):108-12.

44. Eagle H. The specific amino acid requirements of a human carcinoma cell (strain HeLa) in tissue culture. J Exp Med. 1955;102(1): $37-48$.

45. Pirkmajer S, Chibalin AV. Serum starvation: caveat emptor. Am J Phys Cell Phys. 2011;301(2):C272-9.

46. Degenhardt K, Mathew R, Beaudoin B, Bray K, Anderson D, Chen $\mathrm{G}$, et al. Autophagy promotes tumor cell survival and restricts necrosis, inflammation, and tumorigenesis. Cancer Cell. 2006;10(1): 51-64.

47. Shiraishi T, Verdone JE, Huang J, Kahlert UD, Hernandez JR, Torga G, et al. Glycolysis is the primary bioenergetic pathway for cell motility and cytoskeletal remodeling in human prostate and breast cancer cells. Oncotarget. 2015;6(1):130-43. The authors demonstrate that ATP production via aerobic glycolysis, but not via mitochondrial oxidative respiration, is required for cell motility and cytoskeletal rearrangements. Importantly, while aerobic glycolysis is increased in aggressive and motile cancer cell types, mitochondrial respiration - which supplies the majority of ATP - is unchanged between aggressive and less aggressive types, consistent with the Warburg Effect.

48. Alfarouk KO, Ibrahim ME, Gatenby RA, Brown JS. Riparian ecosystems in human cancers. Evol Appl. 2013;6(1):46-53.

49. Aktipis CA, Maley CC, Pepper JW. Dispersal evolution in neoplasms: the role of disregulated metabolism in the evolution of cell motility. Cancer Prev Res. 2012;5(2):266-75.

50. Zhou M, Chaudhury B, Hall LO, Goldgof DB, Gillies RJ, Gatenby RA. Identifying spatial imaging biomarkers of glioblastoma multiforme for survival group prediction. J Magn Reson Imaging. 2017;46(1):115-23.

51. Alfonso JCL, Talkenberger K, Seifert M, Klink B, Hawkins-Daarud A, Swanson KR, et al. The biology and mathematical modelling of glioma invasion: a review. J R Soc Interface. 2017;14(136): 20170490 .

52. Stephens DW, Brown JS, Ydenberg RC. Foraging: behavior and ecology. Chicago: University of Chicago Press; 2007.

53. Cantor JR, Sabatini DM. Cancer cell metabolism: one hallmark, many faces. Cancer Discovery. 2012;2(10):881-98.

54. Ward PS, Thompson CB. Metabolic reprogramming: a cancer hallmark even Warburg did not anticipate. Cancer Cell. 2012;21(3): 297-308.

$55 . \bullet$ Kotler BP, Morris DW, Brown JS. Direct behavioral indicators as a conservation and management tool. Conserv Behav. 2016;21:307. This review discusses how foraging behaviors (diet choice, patch use and habitat selection) can be used as leading indicators for managing wildlife populations and anticipating future changes in the wildlife's population size. The paper provides detail on the foraging theories and behaviors that we present for cancer cells. At present we do not have the tools to completely measure such foraging behaviors, but this may have more to with a lack of interest than intrinsic technological hurdles. In addition to being a hallmark of cancer, optimal foraging behaviors may also provide leading indicators for cancer prognoses, therapy efficacies and rapid turnaround of information required to adjust therapies accordingly and successfully. 